\title{
SSR mining in oil palm EST database: application in oil palm germplasm diversity
} studies.

\begin{abstract}
This study reports on the detection of additional expressed sequence tags (EST) derived simple sequence repeat (SSR) markers for the oil palm. A large collection of 19243 Elaeis guineensis ESTs were assembled to give 10258 unique sequences, of which 629 ESTs were found to contain 722 SSRs with a variety of motifs. Dinucleotide repeats formed the largest group $(45.6 \%$ ) consisting of $66.9 \% \mathrm{AG} / \mathrm{CT}, 21.9 \% \mathrm{AT} / \mathrm{AT}, 10.9 \% \mathrm{AC} / \mathrm{GT}$ and $0.3 \% \mathrm{CG} / \mathrm{CG}$ motifs. This was followed by trinucleotide repeats, which is the second most abundant repeat types $(34.5 \%)$ consisting of AAG/CTT (23.3\%), AGG/CCT (13.7\%), CCG/CGG (11.2\%), AAT/ATT (10.8\%), AGC/GCT (10.0\%), ACT/AGT (8.8\%), ACG/CGT (7.6\%), ACC/GGT(7.2\%), AAC/GTT (3.6\%) and AGT/ACT (3.6\%) motifs. Primer pairs were designed for 405 unique EST-SSRs and 15 of these were used to genotype 105 E. guineensis and 30 E. oleifera accessions. Fourteen SSRs were polymorphic in at least one germplasm revealing a total of 101 alleles. The high percentage (78.0\%) of alleles found to be specific for either E. guineensis or E. oleifera has increased the power for discriminating the two species. The estimates of genetic differentiation detected by EST-SSRs were compared to those reported previously. The transferability across palm taxa to two Cocos nucifera and six exotic palms is also presented. The polymerase chain reaction (PCR) products of three primer-pairs detected in E. guineensis, E. oleifera, C. nucifera and Jessinia bataua were cloned and sequenced. Sequence alignments showed mutations within the SSR site and the flanking regions. Phenetic analysis based on the sequence data revealed that $\mathrm{C}$. nucifera is closer to oil palm compared to J. bataua; consistent with the taxanomic classification.
\end{abstract}

Keyword: Genetic variation; Transferability; Phenetic analysis; Elaeis guineensis. 